\title{
Improvement of catheter-related outcomes after application of tunneled cuffed hemodialysis catheter insertion without fluoroscopy
}

\author{
Seok Hui Kang, Jun Young Do \\ Division of Nephrology, Department of Internal Medicine, Yeungnam University College of Medicine, Daegu, Korea
}

Received: June 30, 2019

Revised: March 5, 2020

Accepted: March 6, 2020

Corresponding author:

Jun Young Do

Department of Internal Medicine,

Yeungnam University College of

Medicine, 170 Hyeonchung-ro,

Nam-gu, Daegu 42415, Korea

Tel: +82-53-680-3844

Fax: +82-53-654-8386

E-mail: jydo@med.yu.ac.kr
Background: Non-tunneled catheters (NTCs) are used for hemodialysis (HD) in many centers in which fluoroscopy is not easily accessed despite high complication rates and conditions requiring long-term HD. Therefore, here we aimed to evaluate the superiority of catheter-related outcomes after the application of tunneled cuffed catheter (TCC) without fluoroscopy versus unconditioned NTC insertion.

Methods: We divided the participants into two phases: those receiving NTCs between March 2010 and February 2011 (phase I), and those receiving TCCs or NTCs between March 2011 and February 2012 (phase II). Catheter survival, nurse satisfaction, and reasons for catheter removal were analyzed.

Results: Two hundred and sixty patients in phase I and 300 patients in phase II were enrolled in this study. The success rate of TCC insertion was 99.2\%. The catheter survival rate in phase I was $65.5 \%$ at 1 month, while that in phase II was $74.9 \%$ at 1 month ( $p=0.023)$. We compared catheter survival between TCCs and NTCs for all periods regardless of phase. The TCC survival rate was higher than the NTC survival rate $(p<0.001)$. Catheter-associated problems led to catheter removal in 97 patients $(26.6 \%)$ in phase I and 68 patients (18.5\%) in phase II ( $p=0.009)$. Among 14 $\mathrm{HD}$ nurses, all reported being satisfied with manipulation during pre-/post-HD, manupulation during $H D$, and overall. Eleven HD nurses (78.6\%) reported being satisfied with the workload. Conclusion: Compared with unconditional NTC insertion for HD, TCC insertion without fluoroscopy improved the overall catheter survival and nurse satisfaction rates.

Keywords: Central venous catheter; Fluoroscopy; Hemodialysis; Renal dialysis

\section{Introduction}

The incidence and prevalence of end-stage renal disease (ESRD) continue to increase worldwide [1-3]. Hemodialysis (HD) is an established renal replacement therapy for patients with ESRD, and the adequate dialysis requires a vascular access, which is achieved via an arterio-venous fistula or graft for chronic HD. However,
Yoon et al. [4] showed that in $83.6 \%$ of patients initiating $\mathrm{HD}$ a central venous catheter is used as the first way of access in Korea.

HD catheters are divided into two groups according to the presence of cuff, that is, into temporary non-tunneled catheters (NTCs) and tunneled cuffed catheters (TCCs) [5,6]. NTCs are usually inserted into a femoral vein (FV) or an internal jugular vein (IJV), whereas TCCs are usually inserted into an IJV. TCCs

Copyright (C) 2020 Yeungnam University College of Medicine

This is an Open Access article distributed under the terms of the Creative Commons Attribution Non-Commercial License (http://creativecommons.org/licenses/by-nc/4.0/) which permits unrestricted non-commercial use, distribution, and reproduction in any medium, provided the original work is properly cited. 
are superior to NTCs in terms of infection rates, patient discomfort, and inadvertent removal [7]. In addition, the Kidney Disease Outcomes Quality Initiative (KDOQI) guideline recommends that an NTC should only be used in hospitalized patients and for less than 1 week [7].

Proper tip placement in TCCs is important to proper function. Fluoroscopy guidance for tip visualization would be considered mandatory for TCC insertion. However, NTCs may be inserted for $\mathrm{HD}$ in many centers in which fluoroscopy is not easily accessed despite high rates of complications and conditions requiring long-term HD. Therefore, we aimed to evaluate the superiority of catheter-related outcomes after the application of TCC without fluoroscopy versus unconditioned NTC insertion.

\section{Materials and methods}

\section{Ethics statement}

This study was approved by the Institutional Review Board of Yeungnam University Hospital (IRB No: YUH-12-0401-052). The board waived the need for informed consent.

\section{Study participants}

We reviewed the medical records of Yeungnam University Medical Center in Korea to identify all adults ( $>18$ years) who underwent HD catheter insertion between March 2010 and February 2012. In March 2011, our center employed a nephrologist with 2 years of experience with TCC insertion. NTCs were inserted between March 2010 and February 2011, while TCCs or NTCs were inserted between March 2011 and February 2012. HD catheter type was chosen by the nephrologist. Therefore, our study was divided into two phases: phase I (March 2010 to February 2011) and phase II (March 2011 to February 2012). Phases I and II were divided according to the application of TCC without fluoroscopy. In phase I, all HD catheters were inserted by NTC. In phase II, the patients requiring $\mathrm{HD}$ within 1 week were inserted with NTC and patients requiring $\mathrm{HD}>1$ week were inserted with TCC without fluoroscopy. At phase I and II, NTC can be used for longer duration than 1-2 week according to clinical progress. Comparison between NTC and TCC using merged data may be associated with selection bias. Therefore, we have divided the study into two phases. NTCs and TCCs were used in the same manner during the two phases. We also compared the outcomes of NTC versus TCC without fluoroscopy regardless of phase.

\section{Catheter insertion methods}

TCCs were inserted as previously described [8]. All were inserted routinely in a HD unit suite at our institution by two nurses and a nephrologist with 2 years of experience. Informed consent was obtained from all patients before the procedure. Briefly, the right (or left) neck and anterior chest wall were prepped with Betadine, and electrocardiography monitoring was utilized. Under ultrasonographic guidance (SA-8000; Medison, Seoul, Korea), the targeted IJV was punctured with a 21-gauge needle; after the confirmation of good venous return, a 0.018-inch guide wire was inserted (Mini Access Kit; Merit Medical Systems Inc., South Jordan, UT, USA), and a 4F sheath was placed. A pre-packaged Seldinger-type double lumen catheter set (14.5 Fr; Covidien, Mansfield, MA, USA or 14.5 Fr; Medcomp, Harleysville, PA, USA) was used that consisted of a double lumen catheter, two vessel dilators (11 and $13 \mathrm{Fr}$ ), a J-tip guide wire, a 15 Fr peel-away sheath, and a tunneler. A J-tip guide wire was advanced under electrocardiogram monitoring. Two skin incisions were made (access site, exit site). A tunnel was created and the catheter was then placed into the tunnel. Serial tract dilation was then performed to accommodate the peel-away sheath. The catheter was inserted using the peelaway sheath placement technique, and catheter function was checked by saline flushes with a 5-mL B.D syringe. Finally, the catheter tip was confirmed by chest X-ray as located within the heart shadow or heading for the right atrium or ventricle. If the catheter tip did not deviate widely from the heart shadow and HD catheter's function was intact, the catheter was not repositioned. Techniques for NTC were same until it is insertion of a 4F sheath into relevant IJV or FV. A 0.038 -inch guide wire was inserted and vessel dilator was inserted over the guidewire. Finally, HD catheter was inserted through the IJV (11 Fr; Medcomp) or FV (11.5 Fr; Medcomp).

\section{Outcome measurements}

The clinical data collected at the HD catheter insertion included age, disease underlying ESRD, sex, dialysis data, laboratory data, and $\mathrm{HD}$ catheter position/type, that is, NTC by the FV, NTC by IJV, or TCC by IJV. The data collected during follow-up included reasons for catheter removal classified as malfunction, infection, bleeding, neck edema, withdrawal from dialysis, death, follow-up loss, use of another access method (conversion from catheter to arterio-venous fistula, graft, or peritoneal dialysis), self-removal, and kidney transplantation. Malfunction, infection, bleeding, and neck edema were classified as catheter-associated problems. Catheter malfunction was defined as a negative pressure less than -250 $\mathrm{mmHg}$ at a blood flow rate $<300 \mathrm{~mL} / \mathrm{min}$ or frequent pressure alarm during the $\mathrm{HD}$ session for adequate dialysis [7]. A catheter infection was diagnosed if pus, redness, induration, or tenderness was noted within $2 \mathrm{~cm}$ around the catheter exit site or along the 
catheter tunnel. A catheter infection also was diagnosed if catheter-related bacteremia was suspected. Bleeding was diagnosed if the exit or puncture site was oozing and treatment beyond a dressing change was needed. Neck edema was diagnosed if neck edema related to the catheter was suspected after the exclusion of other causes. In cases of catheter malfunction or infectious complications, the catheter was removed when the problems were not resolved by catheter manipulation or antibiotics. Technical success was defined as no procedure-associated complications with the catheter from venipuncture to HD initiation. Catheter survival was defined as the time that elapsed between catheter insertion and removal. Causes of catheter removal, excluding catheter-associated problems, were analyzed as censored data. Dialysis and laboratory data were reviewed at the time of $\mathrm{HD}$ initiation, and dialysis was specified as $\mathrm{HD}$ or continuous renal replacement therapy. Laboratory data included hemoglobin $(\mathrm{g} / \mathrm{dL})$, platelet count $(\mathrm{K} /$ $\left.\mathrm{mm}^{3}\right)$, calcium $(\mathrm{mg} / \mathrm{dL})$, phosphorus $(\mathrm{mg} / \mathrm{dL})$, aspartate aminotransferase (AST; IU/L), alanine aminotransferase (ALT; IU/L), blood urea nitrogen $(B U N ; \mathrm{mg} / \mathrm{dL})$, creatinine $(\mathrm{mg} / \mathrm{dL})$, sodium $(\mathrm{mEq} / \mathrm{L})$, and potassium $(\mathrm{mEq} / \mathrm{L})$.

For all $14 \mathrm{HD}$ nurses who participated in both phases, satisfaction with TCC was assessed by four questions about manipulation during pre-/post-HD, manipulation during HD, workload, and total satisfaction. All 14 nurses responded very satisfied, satisfied, no change, dissatisfied, or very dissatisfied.

\section{Statistical analysis}

Continuous values are reported as mean \pm standard deviation and were compared using the $t$-test. Categorical variables are reported as count and percentage, and were compared using Pearson chisquare test or Fisher exact test. Kaplan-Meier analyses were used to identify the intergroup differences in survival. The $p$-values used for survival curve comparisons were calculated using the logrank test. We performed Cox regression analyses of hazard ratio (95\% confidence interval) for survival. Values of $p<0.05$ were considered statistically significant.

\section{Results}

Two hundred and sixty patients in phase I and 300 patients in phase II were enrolled in this study (Table 1). Sex and age did not differ significantly between groups. There were no significant intergroup differences in dialysis modality, hemoglobin, platelet count, calcium, phosphorus, AST, ALT, BUN, creatinine, or potassium level. Serum sodium levels were higher in phase II than in phase I. A total of $364 \mathrm{HD}$ catheters were inserted in phase I ver-

Table 1. Baseline characteristics

\begin{tabular}{|c|c|c|c|}
\hline Variable & Phase I & Phase II & $p$-value $e^{a)}$ \\
\hline No. of patients & 260 & 300 & - \\
\hline Underlying disease of ESRD (DM) & $123(47.3)$ & $144(48.0)$ & 0.870 \\
\hline No. of catheterizations & 364 & 367 & - \\
\hline Age (yr) & $64.0 \pm 15.5$ & $63.8 \pm 14.3$ & 0.837 \\
\hline Male sex & $143(55.0)$ & $177(59.0)$ & 0.340 \\
\hline Hemoglobin (g/dL) & $9.6 \pm 1.8$ & $9.8 \pm 1.9$ & 0.216 \\
\hline Platelet $\left(\mathrm{K} / \mathrm{mm}^{3}\right)$ & $206 \pm 131$ & $198 \pm 126$ & 0.473 \\
\hline Calcium (mg/dL) & $7.7 \pm 1.2$ & $7.5 \pm 1.0$ & 0.297 \\
\hline Phosphorus (mg/dL) & $5.1 \pm 2.3$ & $5.3 \pm 1.9$ & 0.453 \\
\hline Aspartate aminotransferase (IU/L) & $215 \pm 817$ & $200 \pm 496$ & 0.829 \\
\hline Alanine aminotransferase (IU/L) & $102 \pm 317$ & $103 \pm 252$ & 0.987 \\
\hline Blood urea nitrogen (mg/dL) & $69.7 \pm 39.0$ & $65.0 \pm 39.6$ & 0.186 \\
\hline Creatinine (mg/dL) & $6.0 \pm 3.8$ & $5.8 \pm 3.8$ & 0.589 \\
\hline Sodium (mEq/L) & $135.2 \pm 6.4$ & $136.9 \pm 6.7$ & 0.002 \\
\hline Potassium (mEq/L) & $4.4 \pm 1.0$ & $4.2 \pm 0.9$ & 0.206 \\
\hline Continuous renal replacement therapy & $41(15.8)$ & $46(15.3)$ & 0.887 \\
\hline Type of hemodialysis catheter & & & $<0.001$ \\
\hline Non-TCC (FV) & $176(48.4)$ & $171(46.6)$ & \\
\hline Non-TCC (IJV) & $188(51.6)$ & $76(20.7)$ & \\
\hline TCC (IJV) & 0 & $120(32.7)$ & \\
\hline
\end{tabular}

Values are presented as mean \pm standard deviation or number (\%).

ESRD, end-stage renal disease; DM, diabetes mellitus; TCC, tunneled cuffed catheter; FV, femoral vein; IJV, internal jugular vein.

${ }^{\text {a) }}$ Statistical significances were determined using the t-test for continuous variables and Pearson chi-square test for categorical variables. 
sus 367 in phase II. Of them, 120 TCCs were inserted during phase II (32.7\%). The TCC insertion success rate was $99.2 \%$. One complication occurred during TCC insertion due to J-tip guidewire entrapment within the heart. This case was described in a previous paper [9].

The catheter survival rate during phase I was $65.5 \%$ at 1 month and $50.7 \%$ at 5 months, while that during phase II was $74.9 \%$ at 1 month and $63.7 \%$ at 5 months (Fig. 1). Thus, the catheter survival rate during phase II was significantly higher than that during phase I $(p=0.023)$. Furthermore, TCC survival was significantly greater than NTC survival $(p<0.001)$.

Change in access was the most common cause of catheter removal in both phases ( $24.5 \%$ in phase I, $24.8 \%$ in phase II). However, the catheter removal rate for a catheter-associated problem was greater in phase I (26.6\% in phase I, $18.5 \%$ in phase II; $p=0.009)$. The intervals from insertion to event in phases I and II were $33.4 \pm 74.6$ and $34.9 \pm 58.4$ days, respectively $(p=0.758)$. More specifically, in phase I, the removal rate for a catheter-associated problem was $33.0 \%$ for NTC by FV and $20.7 \%$ for NTC by IJV $(p=0.008)$. In phase II, the corresponding rates were $31.0 \%$ for NTC by FV, $14.5 \%$ for NTC by IJV, and 3.3\% for TCC $(p<0.001)$. Catheters were removed due to patient death in phases I and II in 62 patients (17.0\%) and 80 (21.8\%), respectively $(p=0.103)$. Catheters were removed due to non-catheter-associated problems and catheter-associated problems in 267 patients (73.4\%) and 97 patients (26.6\%) in phase I and in 299 (81.5\%) and $68(18.5 \%)$ in phase II, respectively $(p=0.009)$. Catheter removal rates by catheter-associated problems were lower in phase II than in phase I. Fig. 2 shows the specific causes of catheter removal.

We also analyzed the outcomes according to catheter type regardless of phase (Fig. 3). The catheter survival rate was highest for TCC, while that for NTC (IJV) was higher than that for NTC (FV). There was no significant difference in catheter survival of the same catheter type between phases I and II. The number of catheters removed due to catheter-associated problems were 4 (3.3\%), 39 (20.7\%), 11 (14.5\%), 58 (33.0\%), and 53 (31.0\%) for TCC, NTC (IJV) in phase I, NTC (IJV) in phase II, NTC (FV) in phase $\mathrm{I}$, and NTC (FV) in phase II, respectively $(p<0.001)$. The proportion of removals due to catheter-associated problems for each catheter decreased after the application of TCC without fluoroscopy.

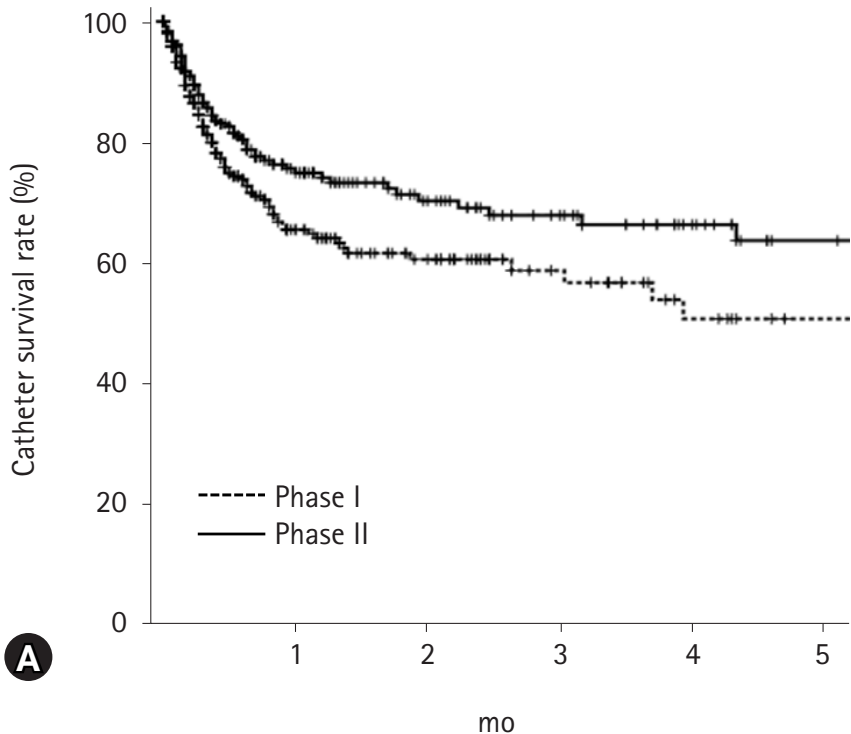

Catheter at risk

$\begin{array}{lrrrrrr}\text { Phase I } & 364 & 96 & 57 & 28 & 15 & 9 \\ \text { Phase II } & 367 & 107 & 65 & 47 & 30 & 18\end{array}$

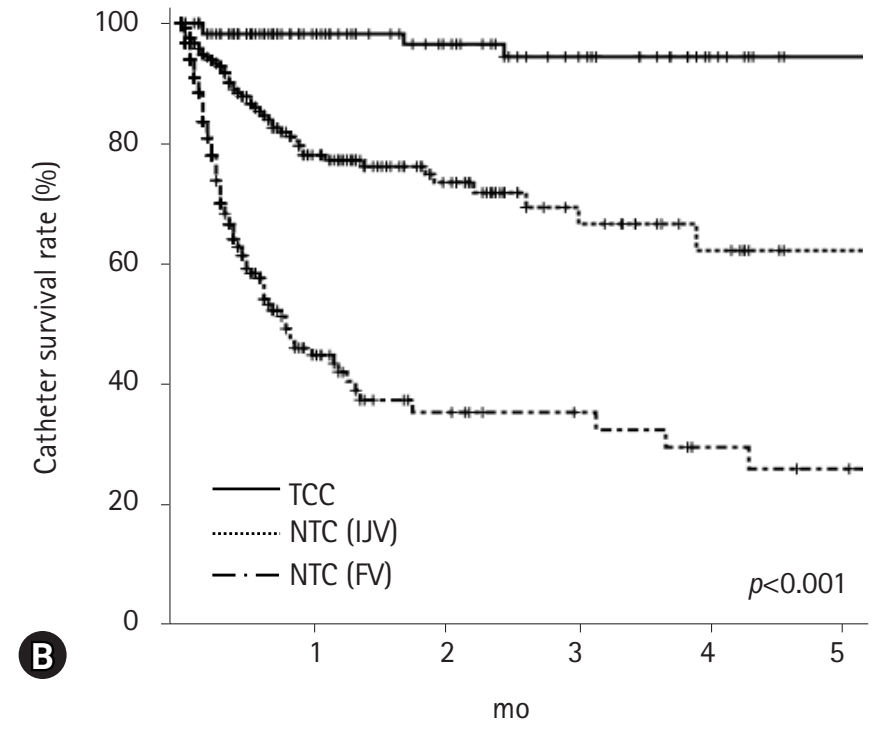

Catheter at risk

$\begin{array}{llllllr}\text { TCC } & 120 & 70 & 52 & 38 & 24 & 15 \\ \text { NTC (IJV) } & 264 & 95 & 53 & 24 & 13 & 6 \\ \text { NTC (FV) } & 347 & 37 & 16 & 12 & 7 & 5\end{array}$

Fig. 1. Catheter survival rate. (A) Plot by application of TCC (phase I, 65.5\% at 1 month and 50.7\% at 5 months; phase II, 74.9\% at 1 month and 63.7\% at 5 months). (B) Plot according to HD catheter type (NTC [FV], 44.7\% at 1 month and 25.6\% at 5 months; NTC [IJV], 77.6\% at 1 month and 61.7\% at 5 months; TCC, 98.2\% at 1 month and 94.4\% at 5 months). TCC, tunneled cuffed catheter; HD, hemodialysis; NTC, non-tunneled catheter; FV, femoral vein; IJV, internal jugular vein. 


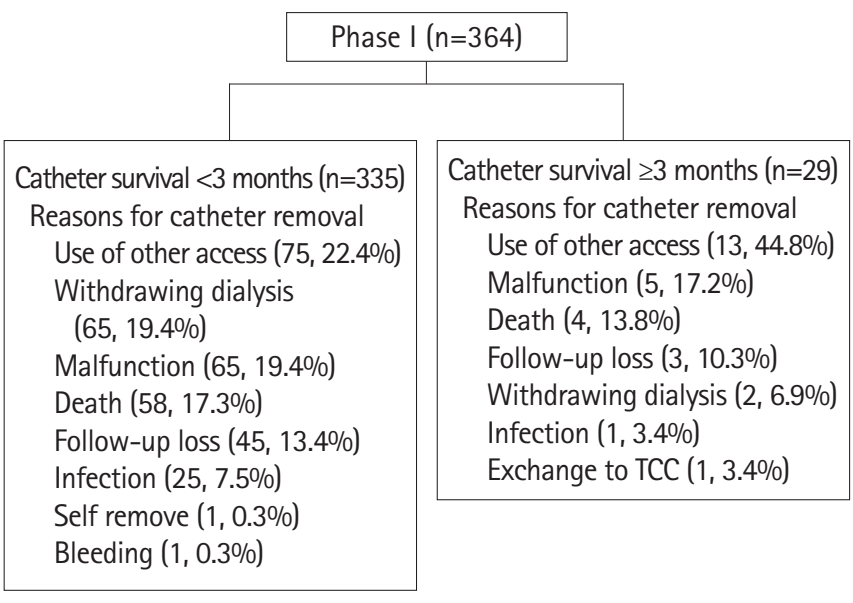

A

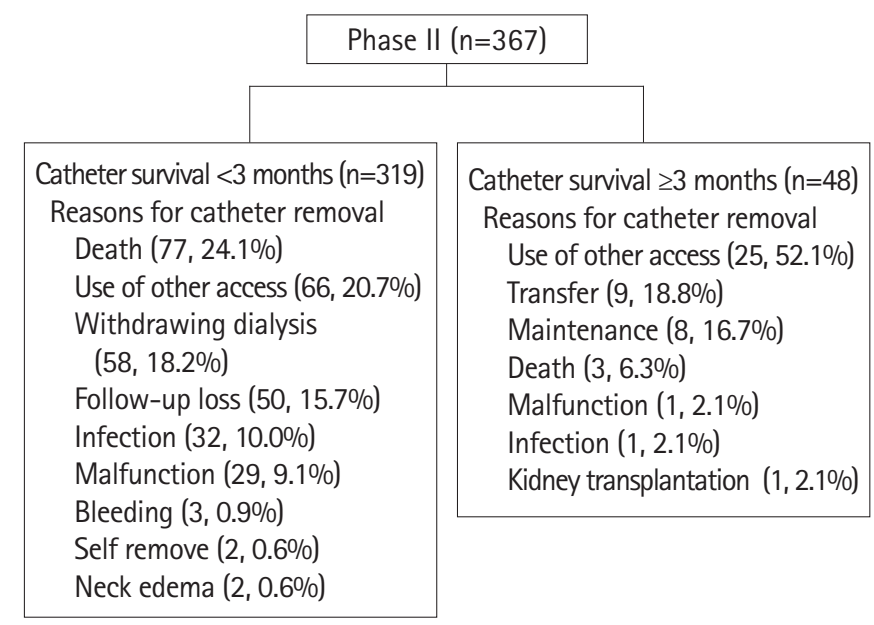

B

Fig. 2. Study population and reasons of catheter removal by study period and catheter survival at 3 months after catheter insertion in (A) phase I, (B) phase II. TCC, tunneled cuffed catheter.

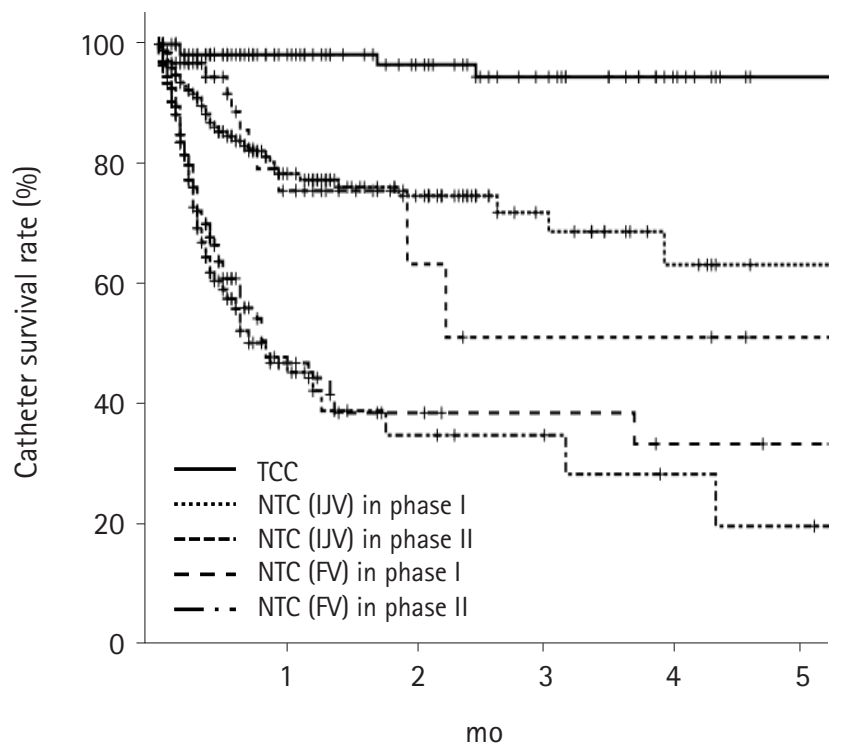

Fig. 3. Catheter survival rates at 5 months for TCC, NTC (IJV) in phase I, NTC (IJV) in phase II, NTC (FV) in phase I, and NTC (FV) in phase II were $94.4 \%, 62.4 \%, 50.0 \%, 31.8 \%$, and $17.8 \%$, respectively. $p<0.001$ for TCC vs. the other catheters, NTC (IJV) in phase I or II vs. NTC (FV) in phase I or II, $p=0.975$ between NTC (IJV) in two phases, and $p=0.494$ between NTC (FV) in phases I and II. TCC, tunneled cuffed catheter; NTC, non-tunneled catheter; IJV, internal jugular vein; $\mathrm{FV}$, femoral vein.

Cox regression analysis, adjusted for diabetes, serum sodium, and old age ( $>65$ years), showed a hazard ratio for catheter survival in phase II of 0.55 (95\% confidence interval, 0.37-0.79; $p=0.002$ ) (Table 2 ). We performed subgroup analyses of age, sex, and diabetes. For elderly, male, or non-diabetic patients, there was a significantly lower risk of catheter removal due to catheter-associated problems in phase II than in phase I (Table 3). We also performed Cox regression analyses for subgroup (Table 4). The multivariate analysis was adjusted for diabetes mellitus, sex, serum sodium level, and phase for the age subgroup; for age, sex, serum sodium level, and phase for the diabetes mellitus subgroup; and for age, diabetes mellitus, serum sodium level, and phase for the sex subgroup. Elderly, diabetics, or male patients showed significantly greater overall catheter survival in phase II than in phase I. The other results showed similar trends, but statistical significance was not observed.

Among the $14 \mathrm{HD}$ nurses, all reported being satisfied with the manipulation during pre-/post-HD, manipulation during $\mathrm{HD}$, and overall. Eleven HD nurses (78.6\%) reported being satisfied with the workload.

\section{Discussion}

Our study showed that the rate of catheter removal due to catheter-associated problems was lower in phase II than in phase I. The both univariate and multivariate analyses showed that the overall catheter survival rate was higher in phase II than in phase I. For elderly, male, or non-diabetic patients, there was a significant lower risk of catheter removal due to catheter-associated problems in phase II than in phase I. For younger, female, or diabetic patients, similar trends were shown, but no statistical significance was observed. For elderly, male, or diabetic patients, catheter survival 
Table 2. Cox regression analyses by variables

\begin{tabular}{|c|c|c|c|c|}
\hline \multirow{2}{*}{ Variable } & \multicolumn{2}{|c|}{ Unadjusted } & \multicolumn{2}{|c|}{ Adjusted } \\
\hline & $\mathrm{HR}(95 \% \mathrm{Cl})$ & $p$-value & $\mathrm{HR}(95 \% \mathrm{Cl})$ & $p$-value \\
\hline Age (ref: $\leq 65$ yr) & $1.30(0.96-1.77)$ & 0.096 & $1.03(0.71-1.47)$ & 0.895 \\
\hline Comorbidity (ref: non-DM) & $1.31(0.96-1.79)$ & 0.085 & $1.28(0.88-1.85)$ & 0.199 \\
\hline Serum sodium (increase 1 unit) & $1.00(0.97-1.03)$ & 0.872 & $1.00(0.97-1.03)$ & 0.979 \\
\hline Phase II (ref: phase I) & $0.70(0.51-0.96)$ & 0.024 & $0.55(0.37-0.79)$ & 0.002 \\
\hline
\end{tabular}

The multivariate analysis was adjusted for age, DM, serum sodium level, and phase.

$\mathrm{HR}$, hazard ratio; $\mathrm{Cl}$, confidence interval; $\mathrm{DM}$, diabetes mellitus.

Table 3. Removal due to catheter-associated problems according to subgroup of age, sex, or diabetes mellitus

\begin{tabular}{lccc}
\hline Variable & Phase I $(n=97)$ & Phase II $(n=68)$ & $p$-value ${ }^{\text {a) }}$ \\
\hline Age $<65 \mathrm{yr}$ & $41(17.6)$ & $30(17.6)$ & 0.120 \\
Age $\geq 65 \mathrm{yr}$ & $56(28.6)$ & $38(19.3)$ & 0.033 \\
Male sex & $56(27.9)$ & $37(17.1)$ & 0.008 \\
Female sex & $41(25.2)$ & $31(20.7)$ & 0.346 \\
Non-diabetes mellitus & $57(28.8)$ & $38(20.1)$ & 0.047 \\
Diabetes mellitus & $40(24.1)$ & $30(16.9)$ & 0.095 \\
\hline
\end{tabular}

Values are presented as number (\%).

${ }^{a)}$ Significant differences between phases were determined using Pearson chi-square test.

Table 4. Cox regression analyses of subgroup of age, sex, or diabetes mellitus

\begin{tabular}{|c|c|c|c|c|}
\hline \multirow{2}{*}{ Variable } & \multicolumn{2}{|c|}{ Unadjusted } & \multicolumn{2}{|c|}{ Adjusted } \\
\hline & $\mathrm{HR}(95 \% \mathrm{Cl})$ & $p$-value & $\mathrm{HR}(95 \% \mathrm{Cl})$ & $p$-value \\
\hline \multicolumn{5}{|l|}{ Age $<65$ yr } \\
\hline Sex (ref: male) & $1.26(0.77-1.95)$ & 0.392 & $1.23(0.72-2.11)$ & 0.443 \\
\hline Serum sodium (increase 1 unit) & $1.00(0.96-1.04)$ & 0.911 & $1.00(0.96-1.05)$ & 0.927 \\
\hline Phase II (ref: phase I) & $0.71(0.44-1.13)$ & 0.144 & $0.65(0.37-1.12)$ & 0.122 \\
\hline \multicolumn{5}{|l|}{ Age $\geq 65 \mathrm{yr}$} \\
\hline Comorbidity (ref: non-DM) & $1.01(0.67-1.53)$ & 0.947 & $0.96(0.57-1.62)$ & 0.885 \\
\hline Serum sodium (increase 1 unit) & $1.00(0.96-1.03)$ & 0.863 & $1.00(0.97-1.04)$ & 0.922 \\
\hline Phase II (ref: phase I) & $0.70(0.46-1.07)$ & 0.097 & $0.49(0.29-0.83)$ & 0.008 \\
\hline \multicolumn{5}{|l|}{ Male sex } \\
\hline Age (ref: $\leq 65$ yr) & $1.53(1.01-2.31)$ & 0.044 & $1.02(0.63-1.66)$ & 0.938 \\
\hline Comorbidity (ref: non-DM) & $1.54(1.01-2.34)$ & 0.044 & $1.43(0.86-2.37)$ & 0.167 \\
\hline Age (ref: $\leq 65$ yr) & $1.05(0.66-1.68)$ & 0.830 & $0.84(0.47-1.49)$ & 0.551 \\
\hline Comorbidity (ref: non-DM) & $1.06(0.67-1.69)$ & 0.799 & $1.12(0.64-1.94)$ & 0.701 \\
\hline Serum sodium (increase 1 unit) & $1.01(0.97-1.06)$ & 0.567 & $1.02(0.97-1.06)$ & 0.476 \\
\hline Phase II (ref: phase I) & $0.88(0.55-1.41)$ & 0.594 & $0.72(0.40-1.30)$ & 0.273 \\
\hline \multicolumn{5}{|l|}{ Non-DM } \\
\hline Sex (ref: male) & $0.85(0.56-1.29)$ & 0.452 & $0.95(0.59-1.56)$ & 0.850 \\
\hline Age (ref: $\leq 65 \mathrm{yr}$ ) & $0.98(0.65-1.47)$ & 0.916 & $0.76(0.48-1.23)$ & 0.268 \\
\hline Serum sodium (increase 1 unit) & $0.98(0.95-1.02)$ & 0.346 & $0.99(0.95-1.02)$ & 0.508 \\
\hline Phase II (ref: phase I) & $0.83(0.55-1.25)$ & 0.369 & $0.68(0.41-1.11)$ & 0.125 \\
\hline \multicolumn{5}{|l|}{$\mathrm{DM}$} \\
\hline Sex (ref: male) & $1.26(0.79-2.02)$ & 0.332 & $1.24(0.71-2.19)$ & 0.450 \\
\hline
\end{tabular}

The multivariate analysis was adjusted for DM, sex, serum sodium level, and phase for the age subgroup; for age, sex, serum sodium level, and phase for the DM subgroup; and for age, DM, serum sodium level, and phase for the sex subgroup.

$\mathrm{HR}$, hazard ratio; $\mathrm{Cl}$, confidence interval; DM, diabetes mellitus. 
rates were higher in phase II than in phase I. For younger, female, or non-diabetic patients, catheter survival was favorable in phase II, but statistical significance was not observed. Nurse satisfaction rates were higher in phase II than in phase I.

Fluoroscopy is not always available at $\mathrm{HD}$ centers because it is expensive and requires materials to protect staff from radiation exposure. TCCs have been inserted in many centers by interventional radiologists familiar with fluoroscopy. TCCs are now being inserted by interventional nephrologists who can perform the procedures necessary for access management. We wanted to evaluate the superiority of TCC without fluoroscopy versus unconditioned NTC, even when fluoroscopy was not available. Catheter outcomes of TCC are undoubtedly better than those of NTC. Oliver reviewed $\mathrm{HD}$ catheter infections and showed a higher risk in NTC versus TCC [10]. Wang et al. [11] investigated 865 dialysis patients and demonstrated a 3.49 odds ratio for catheter dysfunction in NTC versus TCC on multivariate analysis. Mendu et al. [12] showed that TCC had improved dialysis delivery and lower mechanical complication rates than NTC. In our study, 4 (3.3\%) and 161 (26.4\%) TCC and NTC catheters were removed due to catheter-associated problems, respectively $(p<0.001)$. However, NTC may be used for HD in many centers in which fluoroscopy is not easily accessed despite high complication rates of NTC and conditions requiring long-term $\mathrm{HD}$.

In our hospital, all patients requiring $\mathrm{HD}$ were inserted using NTC regardless of the needs for long-term catheterization during phase I. In phase II, although fluoroscopy was not available, we try to use TCCs for patients requiring relatively long-term HD. The success rate of TCC insertion without fluoroscopy was high and only one immediate catheter insertion-related complication was observed. In addition, TCC insertion without fluoroscopy significantly improved the rates of overall catheter survival and catheter removal due to catheter-associated problems. Fluoroscopy is usually used to identify catheter tip position and prevent arterial catheter insertion or puncture. A previous study compared TCC insertion with or without fluoroscopy and found that TCC insertion without fluoroscopy was associated with reduced immediate success rate [13]. However, our study showed favorable results for TCC insertion with versus without fluoroscopy and that the application of TCC without fluoroscopy improved overall catheter survival, catheter-associated complication, and nurse satisfaction rates versus unconditioned NTC insertion.

The KDOQI guideline recommends that TCC be used in patients requiring dialysis for longer than a week and that TCCs should be inserted in centers in which ultrasonography and fluoroscopy are available [7]. However, most patients requiring emergent $\mathrm{HD}$ cannot be withdrawn from $\mathrm{HD}$ within 1 week therefore, most HD catheters used for emergent HD are a TCCs. A study using a NTC showed that the mean dialysis session was $11.3 \pm 6.8$ and catheters were left in place for a mean $19.5 \pm 15.3$ days [14]. Beathard et al. [15] reported 1,765 cases of TCC placement and 2,262 of TCC exchange. The success rates were $98.24 \%$ and $98.36 \%$, respectively, and the complication rates were $1.42 \%$ and $1.41 \%$, respectively. Motta Elias et al. [16] reported their single-center experiences with 130 catheter exchanges from NTC to TCC without fluoroscopy. In their study, the catheter survival rate was $68.0 \%$ at 120 days and the catheter removal rate for a catheter-associated problem was $37 \%$. However, although this previous study was prospective, it involved a single arm and only catheter exchange from NTC to TCC. Furthermore, catheter survival was lower in the in this previous study than in the present study. In the present study, the study period were divided into two phases and the TCC survival rate at 5 months was $94.4 \%$.

This study is limited by its retrospective and single-center nature. Furthermore, we could not evaluate complications during $\mathrm{HD}$ regardless of catheter removal. In addition, all TCCs were inserted by a nephrologist, as practitioner skill can obviously affect complication rates. Therefore, we suggest the need for a larger prospective multi-center study.

In summary, our study showed that TCC insertion without fluoroscopy can be performed with high success rates. Compared with unconditional NTC insertion for HD, the application of TCC insertion without fluoroscopy improved the overall catheter survival and nurse satisfaction rates. Although fluoroscopy was not accessible, TCC insertion without fluoroscopy would help improve catheter-related outcomes.

\section{Acknowledgments}

\section{Conflicts of interest}

No potential conflict of interest relevant to this article was reported.

\section{Funding}

This study was supported by the National Research Foundation of Korea (NRF) grant funded by the Korea government (Ministry of Science and ICT) (2017R1C1B5077075).

\section{Author contributions}

Conceptualization: SHK, JYD; Data curation, formal analysis, and funding acquisition: SHK; Investigation, methodology, software and supervision: JYD; Writing-original draft: SHK; Writing-review \& editing: JYD. 


\section{ORCID}

Seok Hui Kang, https://orcid.org/0000-0003-1023-0195

Jun Young Do, https://orcid.org/0000-0002-6360-9310

\section{References}

1. US Renal Data System. USRDS 2011 annual data report: atlas of chronic kidney disease and end-stage renal disease in the United States [Internet]. Bethesda (MD): National Institutes of Health, National Institute of Diabetes and Digestive and Kidney Diseases; 2011 [cited 2019 May 30]. http://www.usrds.org/ adraspx.

2. McDonald S, Excell L, Livingstone B. ANZDATA: Australia and New Zealand dialysis and transplant registry [Internet]. Adelaide (AU): ANZDATA Registry; [cited 2019 May 30]. http://www.anzdata.org.au/anzdata/.

3. Jin DC. Current status of dialysis therapy in Korea. Korean J Intern Med 2011;26:123-31.

4. Yoon HE, Chung S, Chung HW, Shin MJ, Lee SJ, Kim YS, et al. Status of initiating pattern of hemodialysis: a multi-center study. J Korean Med Sci 2009;24(Suppl 2):S102-8.

5. Vats HS. Complications of catheters: tunneled and nontunneled. Adv Chronic Kidney Dis 2012;19:188-94.

6. Ponikvar R. Hemodialysis catheters. Ther Apher Dial 2005; 9:218-22.

7. Vascular Access Work Group. Clinical practice guidelines for vascular access. Am J Kidney Dis 2006;48(Suppl 1):S248-73.

8. Hwang HS, Kang SH, Choi SR, Sun IO, Park HS, Kim Y. Comparison of the palindrome vs. step-tip tunneled hemodialysis catheter: a prospective randomized trial. Semin Dial 2012; 25:587-91.

9. Kang SH, Park WK, Do JY, Cho KH, Park JW, Yoon KW. J-tip guide wire entrapment within the heart during central venous catheterization. Hemodial Int 2012;16:438-40.

10. Oliver MJ. Acute dialysis catheters. Semin Dial 2001;14:432-5.

11. Wang K, Wang P, Liang X, Lu X, Liu Z. Epidemiology of haemodialysis catheter complications: a survey of 865 dialysis patients from 14 haemodialysis centres in Henan province in China. BMJ Open 2015;5:e007136.

12. Mendu ML, May MF, Kaze AD, Graham DA, Cui S, Chen ME, et al. Non-tunneled versus tunneled dialysis catheters for acute kidney injury requiring renal replacement therapy: a prospective cohort study. BMC Nephrol 2017;18:351.

13. Yevzlin AS, Song GU, Sanchez RJ, Becker YT. Fluoroscopically guided vs modified traditional placement of tunneled hemodialysis catheters: clinical outcomes and cost analysis. J Vasc Access 2007;8:245-51.

14. Yeum CH, Kim SW, Nah MY, Ma SK, Ko JH, Kim NH, et al. Percutaneous catheterization of the internal jugular vein for hemodialysis. Korean J Intern Med 2001;16:242-6.

15. Beathard GA, Litchfield T; Physician Operators Forum of RMS Lifeline Inc. Effectiveness and safety of dialysis vascular access procedures performed by interventional nephrologists. Kidney Int 2004;66:1622-32.

16. Motta Elias R, da Silva Makida SC, Abensur H, Martins Castro MC, Affonso Moyses RM, Pereira BJ, et al. Insertion of tunneled hemodialysis catheters without fluoroscopy.J Vasc Access 2010;11:138-42. 